\title{
A novel method shaping the future of oral hygiene
}

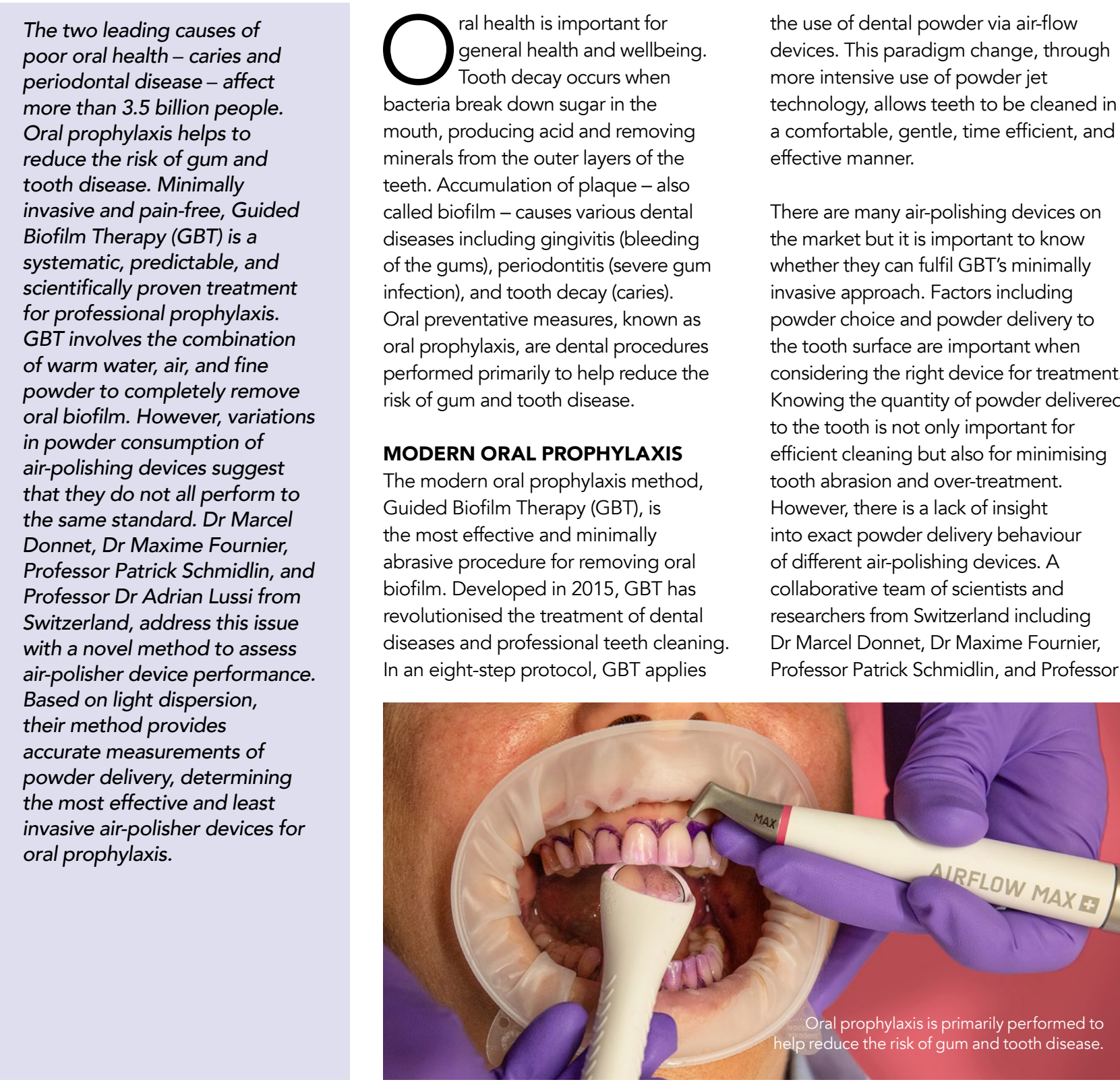

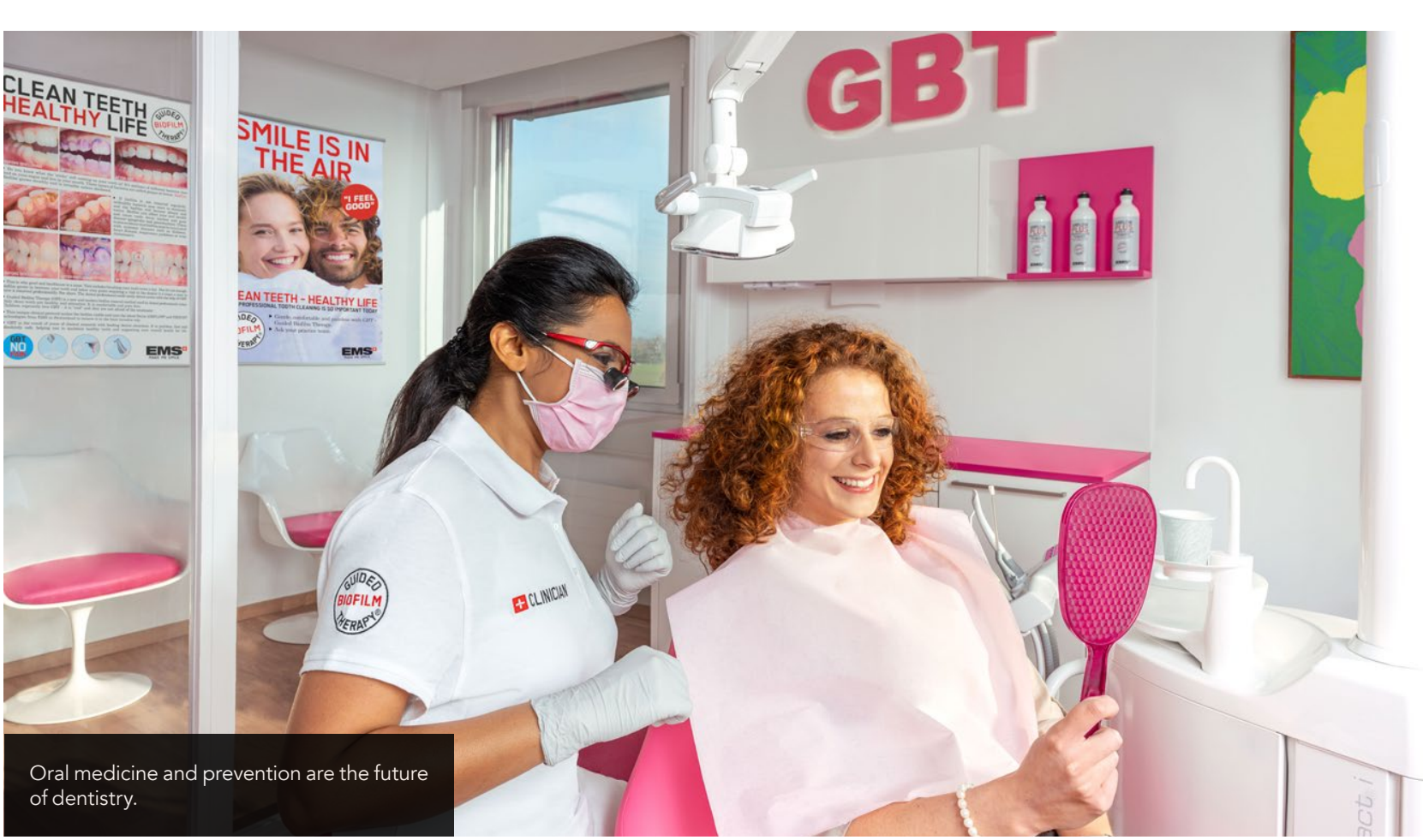

Dr Adrian Lussi, set out to design a method to help understanices mostly for the dentist, allowing thus helping to make the right choice to evaluated in low pressure and gentle perform GBT.

\section{MEASURING POWDER}

CONSUMPTION

The team based its method on light dispersion, which occurs as a powder passes through a beam of laser light. Importantly, the team established the relationship between light intensity cond the quantity of powder (ie, powder consumption). A spectrometer records dilluminated by a bser sour powder mode, representative of an advanced minimally invasive procedure. Powder consumption tests were performed ten times randomly over a three-week period for statistical analysis.

Overall, the table-top devices had more regular flow rates and longer autonomies than the handy devices due to their larger powder chambers. (table-top E1 device) had the longest

The method is based on light dispersion, which occurs as a powder passes through a beam of laser light.

measurements are obtained from the total amount of powder passing through the system, recorded by weighing the and after the test.

\section{AIR-POLISHING}

DEVICE VARIABILITY

Using the new method, six air-polishing devices were compared. three table-top devices for the hygienist) and three autonomy with the most regular powder consumption, offering a slow de E1 device (Handy 3.0 Perio) had the longest autonomy compared to other fond teves. Notably, the researchers the powder chang behaviour inside va pow the most

All devices demonstrated significant variability in the intensity of powder burst at the beginning of each pedal press interval. This was caused by (Combi Touch) had a high powder burst (> 8g/min) compared to the AIRFLOW Prophylaxis Master $(>2 \mathrm{~g} / \mathrm{min})$. This intense burst is clinically important as it can cause an over-delivery of dental powder and subsequent overlay of this may result in inefficient cleaning, reducing the clinical effectiveness of treatment. It can also lead to high powder consumption and powder

Device variability is also a result of the powder behaviour within the powder mer from its start-up time to the stabilisation phase (0.5-5 seconds). An ideal device achieves constant powder consumption during one pedal press. This powder consumption stabilisation was achieved faster in handy devices five seconds all devices reached a steady state, but only in handy E1, and table-top E1 and M3 did this result in regular mean powder consumption.

Other devices showed a decrease in mean powder consumption over consumption In oks of high powder devices will show an over-consumption tubing. For example, the table-top M3 residue on the tooth surface. In turn, 


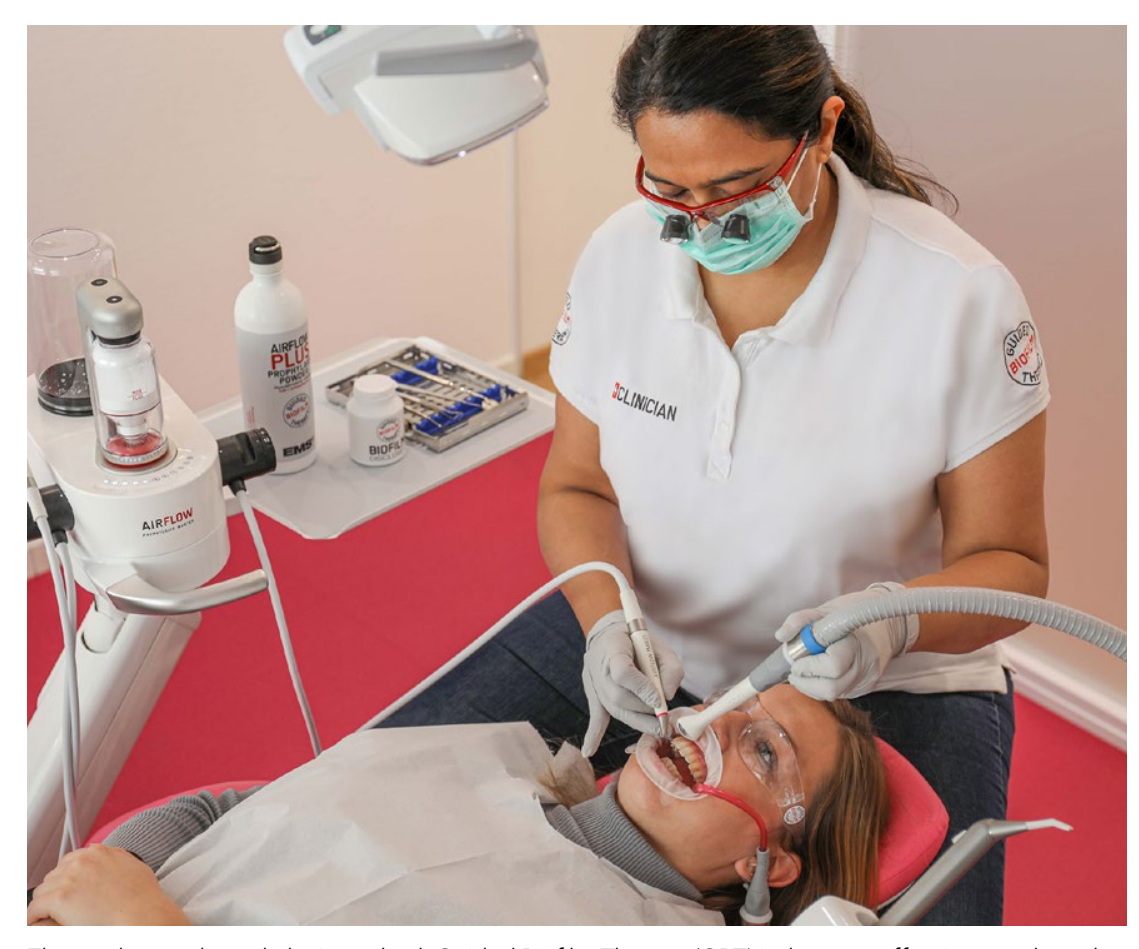

The modern oral prophlyaxis method: Guided Biofilm
least abrasive procedure for removing oral bifilim.

at the beginning of each pedal press. Only table-top E1 and M3 do not have this behaviour.

powder delivery was measured over time due to decreased volume in the powder chamber. Furthermore, the

Different mechanisms employed by the devices to expel powder also affect powder consumption. The

AIRFLOW Prophylaxis Master is the only device that uses a 'venturi' powder principle, whereby powder is taken from the bottom of the chamber. This not well-controlled. had extremely high powder consumption. As a result, this device over-consumes powder and generates more dust as the

powder delivery by speeding up, making evice showed controlled behoviour

The optimisation of air-polisher powder consumption is key to providing best oral prophylaxis.

with a steady powder consumption. ensuring high reproducibility between instantaneous powder consumption, making it easy to use since its performance is predictable. This is purely due to the working mechanism as the decrease in powder volume does not affect the resulting powder consumption.

In contrast, the other devices use 'swirling' pick-up method, whereby powder is taken from the top of the powder chamber. This method was and repeatable, since a decrease in regular refills. that as the po working mecchan consumption consequently decreases. This could result in a change in device chamber empties.
This was seen in the table-top $\mathrm{M} 3$, whic
Pr the device working mode can han to identify the best device for the specified treatment, avoid unpredictable behaviours, and ensure that treatment efficiency remains unaffected. A precise and stable flow rate of dental powder is vital for cleaning sensitive areas including exposed dentine. Indeed, inconsistency in powder delivery to delicate tooth surfaces causes over-treatment and significant damage to these sites during high consumption peaks.

GBT treatment also includes the with powder using a special subgingiva treatment time is 5 seconds powder these results, to enhance the treatment efficiency, a new recommendation time of 7 seconds would ensure the best treatment using the table-top E1. In conclusion, this study helps to understand the working behaviour and subsequent powder delivery of during start-up and during treatment. The study findings may help to prevent over-treatment and adverse impacts on

THE FUTURE OF PREVENTION Oral medicine and prevention are the air-polisher powder consumption is key research highlights the huge differences in powder consumption of different air-polisher devices, which is vital for performing pleasant GBT. It provides important insight into how differences between devices can clinically impact the user and patient treatment.

Only the AIRFLOW` Prophylaxis Master, developed by the EMS Research Center in collaboration with scientists and clinicians, was shown to carry out precise powder delivery. This device guarantees a high-quality GBT treatment, with predictable and repeatable behaviour, while delivering best-in-class results. Due to its superior air-polishing performance, this quality stand treatment of deeper periodontal pockets projection per pocket. According to devices with intense outbursts, both future of dentistry. The optimisation of

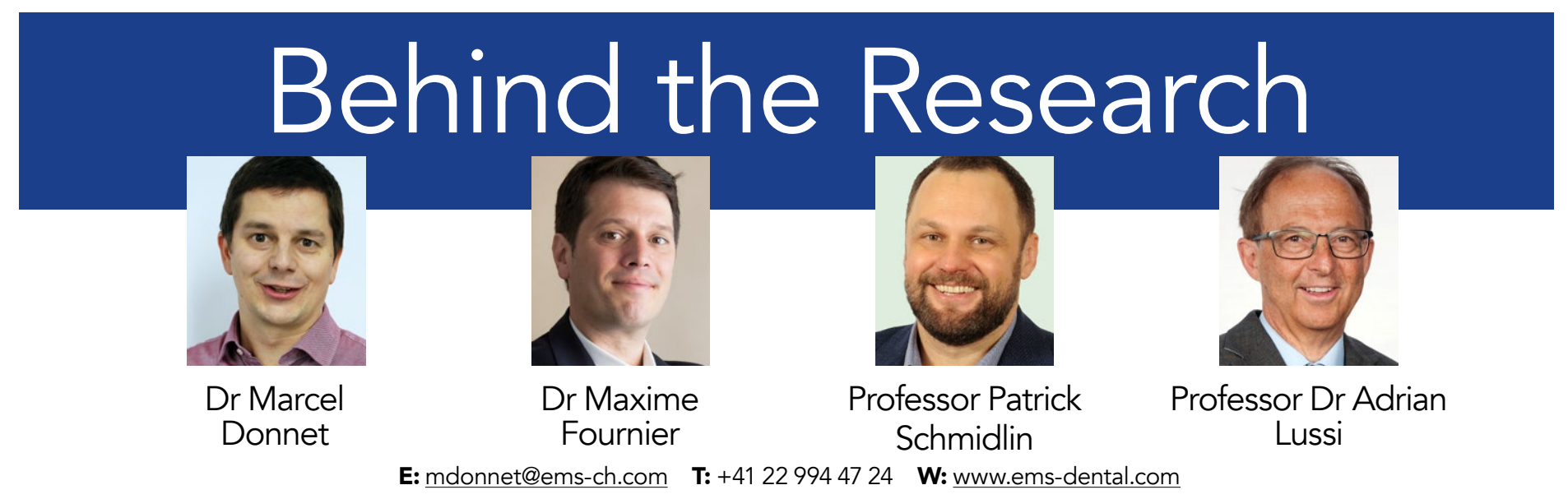

Research Objectives

A novel method was developed to better understand the performance characteristics of air-polishing devices for effective and minimally invasive oral prophylaxis.

\section{Detail}

\section{Address}

EMS Electro Medical Systems SA

Vuarpillière 31, CH-1260 Nyon

Bio

Dr Marcel Donnet is a chemical engineer with a PhD in Powder Technology. He than 20 years in interdisciplinary domains including powders, fluids, chemical, medical, and dental technologies. His passion is to find the best clinical option for both user and patient.

$$
\begin{aligned}
& \text { Dr Maxime Fournier is a human- } \\
& \text { centered technologist with a strong } \\
& \text { passion for disruptive improvement of } \\
& \text { patients' and clinicians' lives. He has } \\
& \text { a PhD in Bioengineering and focused } \\
& \text { his career on pioneering MedTech } \\
& \text { challenges. He is currently the Head of } \\
& \text { Technology and Innovation at Electro } \\
& \text { Medical Systems SA. } \\
& \text { Patrick Schmidlin is Professor and Head } \\
& \text { of the Division of Periodontology and }
\end{aligned}
$$

of Dental Medicine at the University of Zurich. He is a passionate clinician and researcher.

Professor Dr Adrian Lussi holds Master's degree in Chemical Profeering, and DrMed Dent, (CH) and Freiburg (D) He has over 500 publications and is editor or over 500 of 15 books in prevention and operative dentistry. He holds several awards in Dentistry.

\section{References}

Donnet, M, Fournier, M, Schmidlin, PR, Lussi, A, (2021) A Novel Method to Measure the Powder Consumption of Dental Air-Polishing Device, $A$ P
org/10.3390/app11031101

Petersilka, G, Koch R, Vomhof A, et al, (2020) Retrospective ( in supportive periodontal therapy. J Clin Periodontol 48(2) 263-271. doi.org/10.1111/jcpe.13392 and the user potentially expriencing

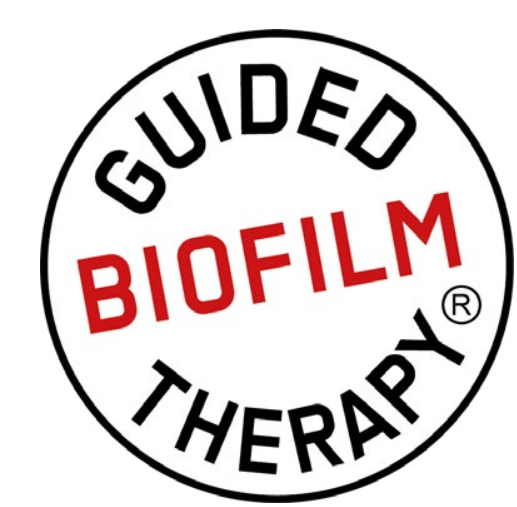

\section{Personal Response}

Could the results of this research change the

II Prevention is better than cure! This applies in particular to dentistry: modern prevention can not only prevent caries, gingivitis, and periodontitis, it can also make a significant contribution to our health more generally (for example Good health begins in the mouth!

Prevention focuses on the removal of pathogenic management today is achieved with AIRFLOW' technology The fine-tuning of powderwaterjet devices is the key to success here stability. Only continuous powder delivery onces perfect biofilm removal. Our method shows that only EMS devices (eg, the AIRFLOW® Prophylaxis Master) can provide optimal biofilm removal with the required controlled powder delivery This quality standard is now called AIR-FLOWING manufacturing of air-polishing devices in the future? dental plaque (biofilm management). The best biofilm

Oar developed an innovative method to 